\title{
Urgences
}

\section{S'en aller sur ses quinze ans au restaurant des regards en coin}

\section{Gilbert Dupuis}

Numéro 12, 3e trimestre 1984

Spécial humour

URI : https://id.erudit.org/iderudit/025183ar

DOI : https://doi.org/10.7202/025183ar

Aller au sommaire du numéro

Éditeur(s)

Urgences

ISSN

0226-9554 (imprimé)

1927-3924 (numérique)

Découvrir la revue

Citer ce document

Dupuis, G. (1984). S'en aller sur ses quinze ans au restaurant des regards en coin. Urgences, (12), 31-38. https://doi.org/10.7202/025183ar d'utilisation que vous pouvez consulter en ligne.

https://apropos.erudit.org/fr/usagers/politique-dutilisation/ 


\section{GILBERT DUPUIS}

\section{S'en aller sur ses quinze ans au restaurant des regards en coin}




\section{S'EN ALLER SUR SES QUINZE ANS AU RESTAURANT DES REGARDS EN COIN}

Ce doit être juin 196... et la neige achève de fondre sur les falaises rouges du littoral de la Baie-des-Chaleurs. Des colonies de pinsons et de fauvettes peuplent déjà les champs de pissenlits et les boisés d'épinettes de Ste-Olivine. C'est le temps de laver les rideaux de l'hiver et de planter un jardin en avant de la grange.

C'est samedi au village plus qu'ailleurs et le samedi repose de l'école et de l'apprentissage de la vie dans les livres. L'algèbre ne rescousse alors plus les filles pour leurs équations à deux beaux inconnus... et les garçons s'entendent bien pour limiter leurs ardeurs à la géométrie dans l'espace des premières... Ils récitent déjà les capitales des cinquante-deux états américains, ils connaissent par coeur les fleuves de France, ils tutoient sur demande tous les volcans de l'Amérique centrale mais ils ne savent pas encore de quoi vivent les habitants de la Matapédia, ni ce qui a poussé les femmes et les hommes de Ste-Bernadette-de-Pellegrin à s'installer dans ces talles de noisettes en pente rocheuse, à vingt milles en arrièrepays de la Baie...

Normandise a treize ans dans les hanches et dix-huit ans dans les yeux. Toute la journée du samedi, elle partage les travaux de sa mère en digne aînée de la famille à douze têtes. Elle apprend son rôle sur le bout de ses doigts brisés contre le plancher, elle accroche les couleurs quotidiennes à la corde à linge, elle redoute que la solidarité des femmes grandisse seulement dans le coeur à l'ouvrage, elle paye sa journée de congé comme tout le monde... La jeune fille a la bonne humeur facile, elle interpelle les rouges-gorges aux fenêtres, elle chan- 
tonne pour s'oublier et perd de grands bouts de corvée dans le scénario rêvassé du samedi soir qui vient. Normandise a treize ans dans les hanches et Raviluc dans la tête...

Le tout jeune homme a cordé de la slabe en avant-midi et fendu des bûches de bois sec après dîner. Raviluc a quatorze ans dans les yeux et déjà vingt ans dans les bras. Toute la journée du samedi, il relève son père pour expier ce jour de relâche scolaire et pour rembourser en son for intérieur ses vingt et un repas de la semaine. L'adolescent se fend en quatre pour sa tranquillité d'esprit et pour abattre l'appréhension grandissante qui monte en lui, au fur et à mesure que le samedi soir s'amène. Raviluc a quatorze ans dans les yeux et Normandise dans la peau...

Et le samedi soir, dès après le souper, c'est la cérémonie du paon pavané dans le miroir de la cuisine d'été. Près de la pompe à bras, Raviluc inventorie ses boutons pubertaires, torture son cuir chevelu, grimace à son visage lunaire, maquille ses dents au soda à pâte, étire et couche son collet sur ses épaules et noircit au charbon les cinq poils et demi que le $V$ de sa chemise ouverte découvre. II ceinture son pantalon, il bretelle sa ceinture, il calcule que ses poches étroites prennent tout leur petit change pour s'ouvrir à la monnaie plus souvent sonnée que sonnante et crache sur ses souliers patins pour un cirage économique, la pénombre fera bien le reste...

Dans une autre maison de Ste-Olivine, Normandise apprécie sa silhouette de coton jaune dans le grand miroir derrière la porte de la chambre dite des maîtres. Son allure achève d'être gracieuse et son profil commence d'être pointu. L'adolescente a tiré sa queue de cheval juste ce qu'il faut pour mettre en valeur ses yeux bridés... pui elle a donné à son regard des relents de ciel à l'aide du bleu à laver! 
Le rituel des préparatifs prend autant d'importance que le voyage au restaurant, car ce qui importe avant tout pour les garçons et les filles, c'est de partir et d'arriver à l'heure stratégique: ni trop tôt pour l'assurance d'être tout de même remarqués, ni trop tard tout de même pour le risque gênant de se faire remarquer...

Ils arrivent à la minute subtile, sifflotant la même chanson et réchauffant le même désir derrière leur front. Les quasifemmes et les presqu'hommes ne se zieutent l'allure qu'à la dérobée toute la première heure. Normandise et Raviluc sont parvenus presqu'en même temps au perron poussiéreux du restaurant Chez Blanche. Mais ils ne se regardent pas de crainte que le papillotement de leurs yeux ne trahisse le battement de leur coeur. La chute du jour rougit l'horizon et les chauves-souris commencent à voleter en zigzag au-dessus de la balançoire publique et du gros autobus bleu et blanc de Transport Huard. À l'intérieur, les garçons se renvoient déjà les balles numérotées sur un tapis vert billard. Les plus adroits empocheurs jouent à jouer l'air grave et ténébreux; les plus gauches jouteurs s'amusent de farces primaires et de contenance gênée. Au comptoir de prélart ciré, les timides et les perdus d'avance trompent leurs appréhensions en repêchant les oeufs du vinaigre ou en léchant, les yeux dans le beurre, deux boules de crème lisse et glacée! Les filles croisent et décroisent leurs jambes de nylon bleu poudre sur le grand banc de bois qui court au pied du mur. Ou bien, elles vont à tour de rôle monter la garde dans la petite salle des miroirs d'où s'échappent, à chaque entrebâillement de la porte, des chuchottements nerveux et des rires étouffés. Toujours deux par deux, elles froufroutent jusqu'au juke-box et, songeuses, écoutent Françoise Hardy chanter mélancolique pour tous les garçons et les filles de leur âge. Les quatre chevelus chantants de Liverpool, dont toutes les villes parlent, font parfois irruption au parloir électrique sous les doigts d'une plus curieuse. II faut bien que quelqu'un dise une part de leurs malaises et de leurs préoccupations. La musique ne doit pas s'arrêter de respirer et c'est à coups de dix cents qu'on nourrit son poumon artificiel. 
Entretemps, Normandise et Raviluc se sont rapprochés sur le grand banc de bois... mais le jeune homme estime qu'à ce rythme de trois pieds la demi-heure, il ne pourra toucher le bras de Normandise que vers quatre heures du matin... Pour une bonne part de la réunion adolescente, les semailles d'oeillades et de plaisanteries portent fruits vers dix heures: se composent alors quelques duos et beaucoup de quatuors... mais se décomposent par contre en silence infiniment de solitaires... dont Raviluc et Normandise.

Ils se sentent bien tout au long de la soirée, tout au long de l'attente par-dessus le feutre vert et la boîte à musique, ils sentent bien l'atmosphère s'imprégner et s'alourdir de leur sensualité juvénile. Des papillons énergiques naissent et grandissent dans la poitrine des actrices et des acteurs de cette récréation pathétique et mystère. Ils économisent la parole mais ruinent leurs ressources télépatiques pour traduire à l'autre ces éclairs d'absolu qui leur mangent la tête et le ventre. Ils ne trouvent pas dans la science de leurs études la nourriture pour satisfaire tous ces appétits qui leur restent inexpliqués dans la gorge et le regard. Dans l'air chaud de ce théâtre populaire, les fringales de tendresse et de rouge à lèvres transcendent très nettement le goût des patates frites et des Cherry Blossom. Une cascade d'émotions tourneboulantes époustoufflent leur coeur quotidien et la langueur équivoque qui leur électrise le corps leur laisse l'impression nouvelle de participer au grand désarroi cosmique...

Mais les filles gardent leur place, à repasser dans leur tête l'apocalypse charnelle des sermons et des silences paternels rappelle-toé ma fille que les avaleurs maniaques de Pepsi sont ben plus rough que les têteux d'Orangeade Crush... méfie-toé surtout des yeux verts et des bottillons pointus... pis à part de ça ben si vous restez en gang, i'vous arrivera rien - et c'est bien ce qu'elles craignaient! Mais l'amour-propre et la pudeur sont 
des gardes du corps bien plus forts que la police familiale. Par ailleurs, les recommandations maternelles retiennent aussi les garçons - vas-y mon gars mais salis pas ta chemise avec les trop grimées... laisse pas ta soeur s'en v'nir toute seule... pis ton deux piasses ben, ménage lé...

Les voilà lancés à reculons dans la vie. Ils sont des visiteurs polis d'un musée de cire. Ils sont des magasineurs envayés promener au bout d'une laisse ombilicale dans une épicerie pleine de pommes juteuses sous emballage transparent. Ils en savent assez pour savoir qu'ils ne savent rien, mais ils en pressentent trop pour dormir tranquille...

Le temps file et les effluves nocturnes de juin passent abondamment la porte du restaurant du coin. Onze heures moins quart. L'haleine des lilas et le respir des épinettes coupent le souffle aux pousseurs de soupirs pas encore "matchés". Normandise et Raviluc rampent l'un vers l'autre dans leur tête. Dehors, sur le chemin de gravelle, la brise humide tutoie les robes légères et les chemisiers ouverts. Ils glissent lentement dans la nuit, ils mènent leur silhouette blanche en promenade fluide et troublante. Seule la stridulation des criquets perturbe, aux alentours d'un ruisseau, le murmure des promeneuses et des promeneurs. Derrière le comptoir du restaurant, la grosse horloge Coca-Cola marque onze heures et demie. Madame Blanche ferme quelques lumières, s'éclaircit la voix et sur un ton désolé les avertit - vous savez les jeunes qu'on farme à minuit?

Dans la salle déjà plus déserte, quelques rougeauds jurent en enfonçant les baguettes dans les boules folles sur le tapis vert. Les filles regardent l'heure, songeuses, et vont se donner un dernier coup de peigne dans l'eau. La tristesse 
passe mal dans la gorge de cette jeunesse tremblante lancée dans l'imitation des aînés sur le marché féroce des apparences. La tête pleine de directives privées, ils enquêtent pour leur propre chef sur la texture de l'avenir et questionnent au jour le jour, le réseau de leurs alarmes. Sur le grand banc de bois, Normandise et Raviluc sentent une impuissance les submerger. La parole est rare et précieux le silence entre eux. Puis, tout à coup, Raviluc tend à Normandise le Cream Soda de la dernière chance... II flageole devant elle, le geste malaisé et la joue en flammes. Normandise hésite d'abord, finit par avancer la main vers l'offrande puis boit, inquiète, le contenu de la bouteille pour éteindre l'incendie dans sa gorge. Elle lâche avec regret le goulot de la bouteille qui lui donnait au moins contenance et lève les yeux vers lui. Raviluc a les oreilles molles et la poitrine pleine d'hirondelles gratouilleuses. II s'emplit le regard de son visage, il ravale son embarras, il va parler, il parle, il dit - avez-vous hâte aux vacances vous autres?

Normandise jette un regard furtif autour, ne voit personne d'autre qu'elle-même, dit oui... puis merci pour la liqueur. Et c'est tout... c'est beaucoup... mais pas assez car Raviluc reste pris avec tous les mots d'urgence qui faisaient la danse du feu derrière ses tempes et qu'il aurait voulu offrir à la jeune fille. Oui, oui comme il aurait aimé lui dire la ferveur joyeuse que son sourire élargissait en lui, comme il aurait goûté la fraîcheur de ses doigts sur sa fièvre, comme il aurait voulu lui conter son désir de l'embrasser au bord de la rivière à Jos Giroux, comme il aurait souhaité s'ouvrir de ces démangeaisons à sa fermeture-éclair, comme il aurait célébré à tuetête l'énergie bouleversante qu'elle infusait à son audace, comme il aurait aimé lui avouer qu'il avait un peu peur d'elle, et de lui aussi, et de tout ce qu'ils ne savaient pas surtout... Or Normandise l'entendit simplement murmurer, et cela la fit rosir - maudit qu'j'ai eu peur qu'tu partes avec Gérard vers minuit moins quart - Et c'est vraiment beaucoup pour la santé de leurs sourires et le reste de leur nuit. Enfin, madame Blanche achève de laver le comptoir et de remplir le rack à 
bouteilles vides; elle complète ensuite l'obscurité, en éteignant une à une, les lumières du restaurant, désormais désert...

Pour les unes et pour les uns, c'est le premier flirt avec le drame de vivre, la conscience du temps qui bouge en dedans, le sentiment de l'existence apparié au vertige et la douleur ambigüe des premières ecchymoses essentielles à l'âme. Pour les unes et pour les uns, la soirée passe en étoile filante porteuse de promesses lourdes à tenir pour l'âge. Les grands enfants de Ste-Olivine vivent le samedi soir comme une écluse entrouverte sur tous les possibles. Le temps coule dans leurs veines et fait peu à peu sauter la digue qui retenait jadis les jeunes castors, en dehors du grand torrent vital. Toute une faune patauge dans ce siècle, qui se sait issue de la forêt, de I'hiver, de la Baie, du restaurant du coin et des manoeuvres désespérées du samedi soir. L'ombre de la solitude déjà traverse la mémoire de tout un chacun qui sait voir toutes les nuances de la lumière... 